\title{
Operator independent left ventricular function monitoring during pharmacological stress echo with the new peak transcutaneous acceleration signal
}

T Bombardini, E Marcelli, E Picano, B Borghi, P Fedriga, B Garberoglio, G Gaggini, G Plicchi

\begin{abstract}
Background-As the myocardium contracts isometrically, it generates vibrations that can be measured with an accelerometer. The vibration peak, peak endocardial acceleration (PEA), is an index of contractility.

Objective-To evaluate the feasibility of PEA measured by the cutaneous precordial application of the accelerometer sensor; and to assess the usefulness of PEA monitoring during pharmacological stress echocardiography.

Design-Feasibility study.

Setting-Stress echo laboratory.

Patients-34 consecutive patients underwent pharmacological stress (26 with dipyridamole; 8 with dobutamine) and PEA monitoring simultaneously.

Interventions-A microaccelerometer was positioned in the precordial region and PEA was recorded. Dipyridamole was infused up to $0.84 \mathrm{mg} / \mathrm{kg}$ in 10 minutes, and dobutamine up to $40 \mu \mathrm{g} / \mathrm{kg} / \mathrm{min}$ in 15 minutes.

Results-A consistent PEA signal was obtained in all patients. Overall mean (SD) baseline PEA was $0.26(0.15) g\left(g=9.8 \mathrm{~m} / \mathrm{s}^{2}\right)$, increasing to $0.5(0.36) g$ at peak stress $(+0.24 g, 95 \%$ confidence interval (CI) 0.14 to $0.34 \mathrm{~g} ; \mathrm{p}<0.01)$. PEA increased from $0.26(0.16)$ to $0.37(0.25)$ $g$ in the dipyridamole group $(+0.11 \mathrm{~g}, 95 \%$ CI 0.08 to $0.16 \mathrm{~g} ; \mathrm{p}<0.01)$, and from $0.29(0.1)$ to $0.93(0.37) \mathrm{g}$ in the dobutamine group $(+0.64 \mathrm{~g}$, 95\% CI 0.37 to $0.91 \mathrm{~g}$; $\mathrm{p}<0.01)$.

Conclusions-Using precordial leads this method offers potential for diagnostic application in the short term monitoring of myocardial function. PEA monitoring is feasible during pharmacological stress and documents left ventricular inotropic response quantitatively in a non-invasive and operator independent fashion.

(Heart 2001;85:286-289)
\end{abstract}

Keywords: ventricular function; contractility; peak endocardial acceleration; stress echo

The contractile state of the heart can theoretically be defined by the maximum velocity of shortening of unloaded myocardial contractile elements. Changes in the maximum rate of rise of ventricular pressure are highly sensitive to acute changes in contractility. ${ }^{2}$ In an attempt to develop methods of monitoring long term changes in function of the human heart, implantable systems using pressure transducers - which detect changes in right ventricular $\mathrm{dP} / \mathrm{dt}$ - have been developed. ${ }^{3}$ The long term use of pressure sensing devices has various technical limitations, and the long term stability of such sensors remains to be demonstrated. ${ }^{4}$ In an attempt to overcome these limitations, alternative approaches have been developed for direct measurements of myocardial vibrations during isovolumic systole. When the myocardium contracts isometrically, it generates vibrations which have audible components that are responsible for the first heart sound. ${ }^{5-7}$ Both the audible and the inaudible spectrum of these vibrations can be measured with an accelerometer. Recent studies have shown that the peak of these myocardial vibrations (PEA, peak endocardial acceleration) occurring in the isovolumic contraction phase-is an index of myocardial contractility and that its directional changes mirror changes in left ventricular peak $\mathrm{dP} / \mathrm{dt}$ very closely. ${ }^{8}$

Our aims in this study were: first, to evaluate the feasibility of the PEA measurements by cutaneous precordial application of the accelerometer sensor; and second, to assess the usefulness of PEA monitoring during pharmacological stress echocardiography.

\section{Methods}

We used a micromass uniaxial acceleration sensor connected to a standard ECG monitoring electrode with foam tape and solid gel. The ceramic piezoelectric accelerometer has a frequency response up to $1 \mathrm{kHz}$ and a sensitivity of $18 \mathrm{mV} / g\left(g=9.8 \mathrm{~m} / \mathrm{s}^{2}\right.$ ) (fig 1$)$.

The monitoring electrode was temporarily positioned in the mid-sternal precordial region before starting the scheduled stress test. The accelerometer was connected to an external signal amplifier with a frequency range of $0.05-1000 \mathrm{~Hz}$. An analogue peak to peak detector synchronised with the standard ECG scanned the first $100 \mathrm{~ms}$ following the $\mathrm{R}$ wave (fig 2). All the data were collected on a seven channel TEAC FM magnetic tape recorder with a frequency response of dc to $1 \mathrm{kHz}$; analogue data were then digitised using a $4 \mathrm{kHz}$ 


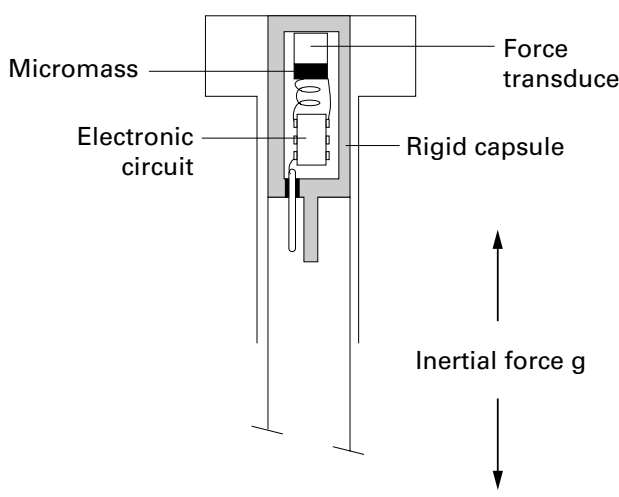

Figure 1 A schema of the micromass acceleration sensor used to sense the peak myocardial acceleration (PEA). The micromass is hermetically sealed inside a non-deformable capsule and needs no external reference or calibration. $g$, acceleration unit $\left(9.8 \mathrm{~m} / \mathrm{s}^{2}\right)$.

sampling rate with 12 bit resolution and stored and processed by an Intel/ 80386 based computer (Santa Clara, California, USA).

All the patients who participated in the study gave informed consent.

Thirty four patients consecutively referred to our stress echo laboratory simultaneously underwent a pharmacological stress echocardiogram (with dipyridamole in 26 patients and dobutamine in eight) and PEA monitoring (with a cutaneous precordial lead). According to standard protocols dipyridamole was infused up to $0.84 \mathrm{mg} / \mathrm{kg}$ in 10 minutes, and dobutamine up to $40 \mu \mathrm{g} / \mathrm{kg} / \mathrm{min}$ in $15 \mathrm{~min}$ utes. ${ }^{9} 10$

The PEA signal was continuously recorded on a magnetic tape and the peak of the acceleration signal (apex to nadir) occurring in the pre-ejection systolic period was automatically measured at baseline, during the test, and at peak stress.

The wall motion score index (WMSI) was calculated in each patient at baseline and peak stress, according to the recommendations of the American Society of Echocardiography, from $1=$ normal to $4=$ dyskinetic in a 16 segment model of the left ventricle. ${ }^{11}$

STATISTICS

Data are expressed as mean (SD). Intragroup comparisons were performed using the paired Student $t$ test. Intergroup comparisons were

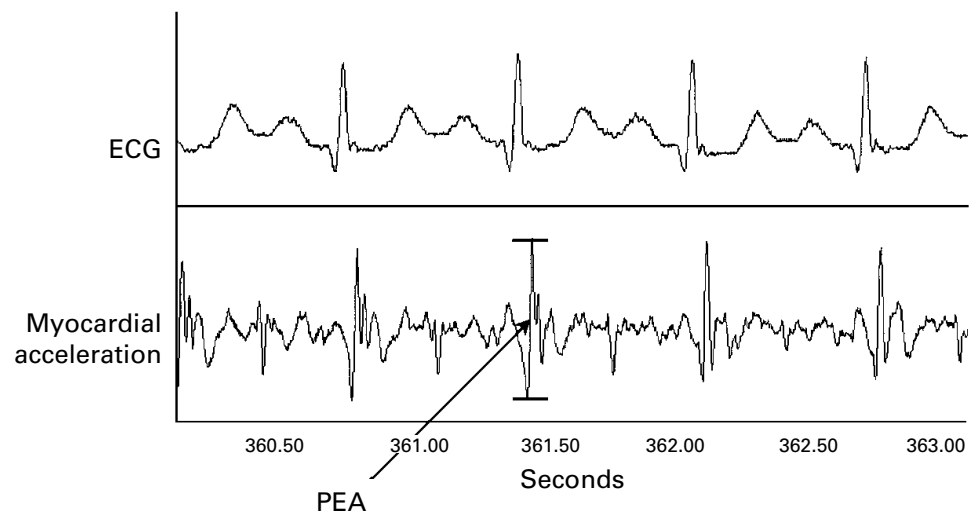

Figure 2 Typical ECG and accelerometer tracings (myocardial acceleration). The peak of the endocardial accelerometer tracing (PEA) occurs during the isovolumic systole, in a 100 ms interval following the ECG $R$ wave.
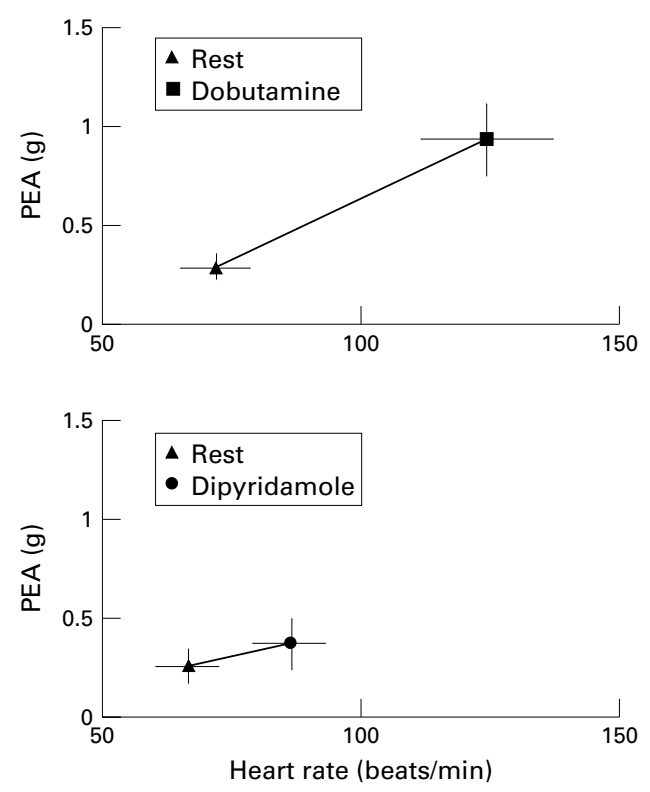

Figure 3 Peak endocardial acceleration signal (PEA) on the $y$ axis in patients who underwent stress

echocardiography with dobutamine (upper panel, eight patients) or dipyridamole (lower panel, 26 patients). Heart rate on the $x$ axis (beats/min). PEA monitoring documented the left ventricular inotropic response quantitatively; it was milder with dipyridamole $(+46$ (38)\%) and more substantial with dobutamine (+235 (107)\%). g, acceleration unit $\left(9.8 \mathrm{~m} / \mathrm{s}^{2}\right)$.

performed using the unpaired $t$ test. When the Levene test was significant at 0.05 , the non-parametric Wilcoxon test for intragroup comparisons and the Mann-Whitney test for intergroup comparisons were performed to confirm significance. Relations between variables were assessed using linear regression analysis and Pearson's correlation coefficient. A probability value of $\mathrm{p}<0.05$ was considered significant.

\section{Results}

STRESS ECHOCARDIOGRAPHY DATA

Of the 26 patients of the dipyridamole group, 15 had normal resting ventricular function (WMSI = 1) and 11 had regional left ventricular motion abnormality (WMSI $=1.5(0.34)$ ). Of the eight patients of the dobutamine group, three had normal resting ventricular function (WMSI = 1) and five had regional left ventricular motion abnormality (WMSI $=1.9$ (0.33)). Heart rate increased from 67 (14) to 87 (13) beats/min after dipyridamole $(+20$ beats/min, $95 \%$ confidence interval (CI) 12 to 28 beats/min; $\mathrm{p}<0.01$ ), and from 73 (16) to 126 (30) beats/min after dobutamine (+53 beats $/ \mathrm{min}, 95 \%$ CI 34 to 72 beats $/ \mathrm{min}$; $\mathrm{p}<0.01)$.

PEA DATA

A consistent PEA signal was obtained in all patients (fig 2). In the patients as a whole, mean (SD) baseline PEA was $0.26(0.15) \mathrm{g}$, increasing to $0.50(0.36) g$ at peak stress $(+0.24 \mathrm{~g}$, $95 \%$ CI 0.14 to $0.34 g ; \mathrm{p}<0.01)$. PEA increased from $0.26(0.16) g$ to $0.37(0.25) g$ in the dipyridamole group $(+0.11 \mathrm{~g}, 95 \%$ CI 0.08 to $0.16 g ; \mathrm{p}<0.01)$, and from $0.29(0.1)$ to $0.93(0.37) \mathrm{g}$ in the dobutamine group 

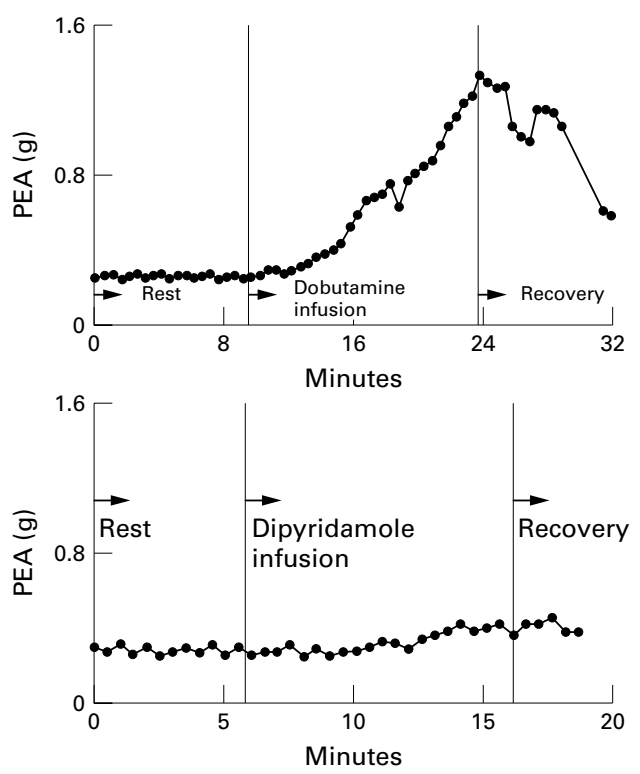

Figure 4 Plot of peak endocardial acceleration signal (PEA) in typical dipyridamole and dobutamine pharmacological stress echocardiograms. Dipyridamole was infused up to $0.84 \mathrm{mg} / \mathrm{kg}$ in 10 minutes and dobutamine up to $40 \mu \mathrm{g} / \mathrm{kg} / \mathrm{min}$ in 15 minutes. PEA increased from 0.28 to $0.42 \mathrm{~g}$ in the dipyridamole study and from 0.27 to $1.32 \mathrm{~g}$ in the dobutamine study. The persistence of inotropic changes in the recovery periods is also shown.

$(+0.64 \mathrm{~g}, 95 \%$ CI 0.37 to $0.91 \mathrm{~g} ; \mathrm{p}<0.01)$. Mean PEA percentage increase was $+46(38) \%$ in the 26 dipyridamole patients and +235 $(107) \%$ in the eight dobutamine patients ( $\mathrm{p}<0.01$ between groups; $95 \%$ CI $100 \%$ to $279 \%$ ) (fig 3).

In the dipyridamole group the PEA increase was similar in the 15 patients with normal resting left ventricular function and in the 11 patients with a regional left ventricular wall motion abnormality at rest $(+45(36) \% v+48$ (43) $\%$, NS; $95 \%$ CI $-35 \%$ to $+29 \%$ ).

In the dobutamine group the PEA increase was greater in the three patients with resting normal left ventricular function than in the five patients with a regional left ventricular wall motion abnormality (+338 (73)\% v +174 $(68) \%, p<0.05 ; 95 \%$ CI $39 \%$ to $288 \%$ ).

In the group of patients as a whole, the PEA percentage increase from baseline to peak stress was significantly related to the percentage increase in heart rate $(r=0.7, \mathrm{p}<0.05)$.

A typical PEA trend during dipyridamole and dobutamine stress is shown in fig 4 .

\section{Discussion}

THE PEA SIGNAL

A stable, reproducible, and consistent PEA signal was obtained in all patients. The signal to noise ratio was high, and little beat to beat variation was observed. Myocardial vibrations show several peculiar time frequency components. An analogue peak to peak detector synchronised with the ECG R wave scanned an appropriate time interval containing the isovolumic contraction phase, and we labelled the maximum value detected during this window PEA. This high amplitude vibration is an expression of the tension wavefront produced during initial activation of the heart. It occurs at the onset of endocardial movement, an average of $20 \mathrm{~ms}$ before mitral valve closure, and was fairly consistent among patients. ${ }^{12-14}$

PROPAGATION OF THE SIGNAL

Baseline PEA value had an ample range (from 0.1 to $0.7 \mathrm{~g}$ ), unrelated to age or left ventricular function. Cardiac vibrations propagate as mechanical shear waves, and the intervening viscoelastic thoracic tissue attenuates the higher frequencies and introduces a variable propagation delay. The absolute PEA value in the single patient can be related more to the transthoracic propagation of cardiac vibrations than to left ventricular function. In fact, when measured epicardially or endocardially, preejection cardiac vibrations are up to 10 times more powerful than when measured on the chest. $^{15-19}$ In a multicentre PEA feasibility study, mean (SD) basal endocardial PEA was $0.45(0.25) g$-more powerful than our precordial PEA values $(0.27(0.14) g){ }^{2021}$

\section{PEA SIGNAL AND INOTROPIC CHANGES}

Pharmacological inotropic stimulation increased the strength of the vibration signal, in keeping with the experimental studies. ${ }^{8}$ It is known that dobutamine increases heart rate and ventricular contractility. Its major characteristic is that it exerts a potent inotropic effect. The inotropic response is co-mediated by $\beta_{1}$ and $\alpha$ adrenoreceptors, the latter causing an inotropic component that is independent of any chronotropic effect. ${ }^{22}{ }^{23}$

Dipyridamole has the well known coronary vasodilator effects mediated by the inhibition of adenosine cellular transport, eventually leading to extracellular adenosine accumulation and steal phenomena. There is a mild catecholamine release that is responsible for the inotropic effect of the drug. ${ }^{9}$ These data are consistent with our findings, where there was a more pronounced and prolonged increase in the PEA signal in the dobutamine group than in the dipyridamole group.

CHOICE OF PHARMACOLOGICAL ECHO STRESS TO TEST THE PEA SIGNAL

For testing the ability of transthoracic PEA to measure cardiac inotropic changes, a cutaneous sensor is the preferred type as it is very sensitive to vibrations from the myocardium. However, it is also sensitive to skeletal muscle vibrations, so during physiological upright bicycle or treadmill testing a considerable amount of noise occurs.

The relative advantages and disadvantages of transthoracic versus endocardial PEA are now clear. Transthoracic PEA is a non-invasive measurement for short term monitoring in resting patients; endocardial PEA is an invasive measurement for long term monitoring with an implantable device, to measure the signal in daily life, in moving or exercising patients, so as to follow the success or adverse effects of chronically administered cardioactive drugs. ${ }^{2021}$ 
THE PEA SIGNAL IN CLINICAL PRACTICE

The clinical applicability of the method using precordial leads offers potential for diagnostic applications in the short term monitoring of myocardial function, to measure spontaneously occurring changes in heart function in patients in bed. It also represents a physiological, operator independent tool for assessing directional global contractility changes during pharmacological stress echo and for post-test inotropic recovery. ${ }^{24}$

CONCLUSIONS

PEA monitoring is feasible during pharmacological stress and documents the left ventricular inotropic response quantitatively in a totally automatic, non-invasive, and operator independent fashion. The response is shown to be milder with dipyridamole and more substantial with dobutamine.

The data obtained in this single centre feasibility study suggest that transcutaneous PEA is a good signal for assessing intraindividual changes in myocardial contractility. To establish fully the advantages and limits of this new method, comparisons with left ventricular pressure-volume loops in humans and multicentre study data are needed.

We thank Mr Keith Smith for his help in correcting the English translation.

1 Gleason WL, Braunwald E. Studies on the first derivative of the left ventricular pressure pulse in man. 7 Clin Invest the left ventricul.

2 Mason DT. Usefulness and limitations of the rate of rise of intraventricular pressure $(\mathrm{dP} / \mathrm{dt})$ in the evaluation of myocardial contractility in man. Am f Cardiol 1969;23:516-27.

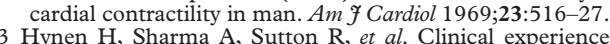
Hynen H, Sharma A, Sutton R, et al. Clinical experience
with VVIR pacing based on right ventricular dP/dt. Eur $f$ Cardiac Physiol Electrophysiol 1991;1:138-46.

4 Lau C-P. Rate adaptive cardiac pacing: single and dual chamber pacing. Armonk, New York: Futura, 1993:169-77.

5 Pantalos G, Kim C, Robinson P, et al. Characterization of natural and total artificial heart acceleration. Trans Am Soc Artif Intern Organs 1989;35:235-8.
6 Wood JC, Festen MP, Lim MJ, et al. Regional effects of mood JC, Festen MP, Lim MJ, et al. Regional effects of myocardial ischemia on epicardially recorded

heart sound. I Appl Physiol 1994;76:291-302.
7 Wood JC, Buda AJ, Barry DT. Time-frequency transforms: a new approach to first heart sound frequency dynamics. IEEE Trans Biomed Eng 1992;39:730-40.

8 Rickards AF, Bombardini T, Corbucci G, et al. An implantable intracardiac accelerometer for monitoring myocardial contractility. PACE 1996;19:2066-71.

9 Picano E, Marini C, Pirelli S, et al. Safety of intravenous high dose dipyridamole echocardiography. The echoPersantin international cooperative study group. Am f Cardiol 1992;70:252-8.

10 Picano E, Mathias WJ, Pingitore A, et al, on behalf of the EDIC study group. Safety and tolerability of dobutaminetropine stress echocardiography: a prospective, large scale, tropine stress echocardiograph: a prospectiv

Picticeno E. Stress echocardiography, 3rd

ed. Heidelberg: Springer-Verlag, 1997:51-6.

12 Yoganathan AP, Gupta R, Udwadia FE, et al. Use of the fast Fourier transform for frequency analysis of the first heart sound in normal man. Med Biol Eng 1976;14:69-73.

13 Adolph RJ, Stephens JF, Tanaka K. The clinical value of frequency analysis of the first heart sound in myocardial infarction. Circulation 1970;41:1003-11.

14 Laniado S, Yellin EL, Miller H, et al. Temporal relation of the first heart sound to closure of the mitral valve. Circulation 1973;47:1006-14.

15 Verburg J. Transmission of vibrations of the heart to the chest wall. In: Ghista DN, Ain A, eds. Advances in cardiovascular physics. Basel: Karger AG, 1989:84-103.

16 Vermarien H. Mapping and vector analysis of heart vibration data obtained by multisite phono-cardiography. In: Ghista DN, Ain A, eds. Advances in cardiovascular physics. Basel: Karger AG, 1989:135-85.

17 Kanai H, Satoh H, Hirose K, et al. A new method for measuring small local vibrations in the heart using ultrasound. IEE Trans Biomed Eng 1993;40:1233-41.

18 Bertrand CA, Milne IG, Hornick RA. Study of heart sound and murmurs by direct heart recordings. Circulation 1956; 13:49-57.

19 Zalter R, Hardy HC, Luisada AA. Acoustic transmission characteristics of the thorax. F Appl Physiol 1963;18:42836.

20 Langenfeld $\mathrm{H}$, Krein $\mathrm{A}$, Kirstein $\mathrm{M}$, et al, for the European PEA Clinical Investigation Group. Peak endocardial pacemaker. PACE 1998;21:2187-91.

21 Clémenty J. Dual chamber rate responsive pacing system driven by contractility: final assessment after 1 -year followup. PACE 1998;21:2192-7.

22 Ross J, Linhart JW, Braunwald E. Efforts of changing heart rate in man by electrical stimulation of the right atrium: rate in man by electrical stimulation of the right atrium: studies at rest, during exe

23 Braunwald E. Assessment of cardiac function. In: Braunwald E, ed. Heart disease, 4th ed. Philadelphia: WB Saunders, 1992:431-4.

24 Weidmann B, Lepique CU, Jansen W, et al. Myocardial infarction as a complication of dobutamine stress echocardiography. $\mathcal{F}$ Am Soc Echocardiogr 1997;10:768-71. 